\title{
Long-term Efficacy of Dacryoendoscopy-guided Recanalization and Silicone Tube Intubation
}

\author{
Sung Eun Kim ${ }^{1}$, Jin Uk Beak ${ }^{2}$, Ji-Sun Paik ${ }^{2}$, Juwan Park ${ }^{3}$, Suk-Woo Yang ${ }^{1}$ \\ ${ }^{1}$ Department of Ophthalmology, Seoul St. Mary's Hospital, College of Medicine, The Catholic University of Korea, Seoul, Korea \\ ${ }^{2}$ Department of Ophthalmology, Yeouido St. Mary's Hospital, College of Medicine, The Catholic University of Korea, Seoul, Korea \\ ${ }^{3}$ ICON Eye Center, Seoul, Korea
}

Purpose: To investigate the long-term efficacy of dacryoendoscopy-guided recanalization and silicone tube intubation in patients with obstruction in the lacrimal drainage system and to identify factors related to surgical outcome.

Methods: We retrospectively reviewed the medical records of patients with primary nasolacrimal duct obstruction and canalicular obstruction who underwent dacryoendoscopy-guided recanalization and silicone tube intubation between August 2014 and March 2016. Factors related to surgical outcome were examined and compared between the success group (eyes with complete response and partial response) and the failure group. Kaplan-Meier survival analysis and multivariable logistic regression analysis were used to analyze the success rate according to the factors found to have statistical significance.

Results: The study included 74 eyes of 51 patients. The mean age of the patients was $60.3 \pm 10.0$ years (range, 34-80 years). The success group consisted of 66 eyes (89.2\%) (complete response, 56 eyes, 75.7\%; partial response, 10 eyes, 13.5\%) and the failure group consisted of eight eyes (10.8\%). The median follow-up period was 58 months (range, 6.5-72 months), and the overall success rate was $89.2 \%$. Compared to the eyes with preoperative lacrimal irrigation test of partial passage, the eyes with no passage were associated with a lower success rate $(95.9 \%$ vs. $76.0 \%, p=0.01)$. Postoperative inflammation was also associated with a lower success rate $(96.6 \%$ vs. $60.0 \%, p<0.001)$.

Conclusions: Dacryoendoscopy-guided recanalization and silicone tube intubation is effective and can be considered a first choice of treatment for eyes which show partial passage in the lacrimal irrigation test. The management of postoperative inflammation is essential to ensure surgical success.

Key Words: Dacryocystorhinostomy; Dacryoendoscopy; Lacrimal endoscopy; Nasolacrimal duct obstruction; Silicone tube intubation

Received: July 22, 2021 Final revision: December 16, 2021

Accepted: December 22, 2021

Corresponding Author: Juwan Park, MD, PhD. ICON Eye Center, 492 Gangnam-daero, HM Tower, Gangnam-gu, Seoul 07345, Korea. Tel: 822-6485-8275, Fax: 82-2-6485-8276, E-mail: parkjw120@gmail.com

Co-corresponding Author: Suk-Woo Yang, MD, PhD. Department of Ophthalmology, Seoul St. Mary's Hospital, College of Medicine, The Catholic University of Korea, 222 Banpo-daero, Seocho-gu, Seoul 06591, Korea. Tel: 82-10-8911-4047, Fax: 82-2-599-7405, E-mail: yswoph@hanmail.net
The advent of dacryoendoscopy, microendoscopy of the lacrimal drainage system, has enabled direct visualization of the canaliculi, common canaliculus, lacrimal sac, and nasolacrimal duct (NLD) [1-4]. The development of dacryoendoscopy has provided a better understanding of the pathology of diseases of the lacrimal drainage system. It can be used not only as a diagnostic tool for the localiza- 
tion and differentiation of lesions such as mucosal edema, stenosis, strictures, stones, and obstructions throughout the lacrimal drainage system [5-7], but also as a therapeutic tool for diseases such as primary nasolacrimal duct obstruction (PNLDO), congenital nasolacrimal duct obstruction (NLDO), and canalicular obstruction [7-9].

Currently, dacryoendoscopic surgery is used as an alternative approach for the treatment of PNLDO. Dacryoendoscopy allows microendoscopic procedures such as guided expansion, laser dacryoplasty, microdrill dacryoplasty, and recanalization of the lacrimal drainage system to be performed with optimal results [10]. Above all, patient satisfaction is high because this procedure restores the integrity of the natural lacrimal drainage system and recovery is fast. However, the reported success rates vary and studies regarding the long-term success rate of this procedure are limited. A recent study by Lee et al. [11] reported a success rate of $87.2 \%$ in 86 eyes (78 with partial NLDO and eight with complete NLDO). However, although the success rate was high, the follow-up period was only 17.2 months. Volkerling et al. [12] studied 215 eyes and reported a success rate of $59.1 \%$ with a median follow-up period of 55 months (range, 31-77 months).

Therefore, in this study, we aimed to examine the value of dacryoendoscopy as a diagnostic tool by comparing it with the conventional diagnostic method and to determine the long-term efficacy of dacryoendoscopy-guided recanalization and silicone tube intubation (STI) in patients with obstruction in the lacrimal drainage system. In addition, we examined the factors related to surgical outcome.

\section{Materials and Methods}

\section{Ethics statement}

The Institutional Review Board of the Catholic University of Korea approved the research protocol (No. KC21RASI0643), and the study was conducted in accordance with the tenets of the Declaration of Helsinki. All patients gave their informed consent.

\section{Study subjects}

We retrospectively reviewed the medical records of patients who underwent dacryoendoscopy-guided recanaliza- tion and STI between August 2014 and March 2016. The obstruction of the lacrimal drainage system was classified according to the location and the extent of the obstruction. The patients were diagnosed with partial canalicular obstruction, complete canalicular obstruction, partial NLDO, or complete NLDO based on a history of continuous epiphora and discharge, diagnostic lacrimal probing and irrigation findings, fluorescein dye disappearance test (FDDT) findings, and dacryoscintigraphy. Epiphora was assessed by a Munk score greater than 1 and the height of tear meniscus $>0.3 \mathrm{~mm}$ (Munk score: 0 , no epiphora; 1 , occasional epiphora requiring wiping less than twice a day $(<2)$; 2, wiping 2-4 times per day; 3, wiping 5-10 times per day; and 4 , wiping $>10$ times per day or continuous tearing). After instillation of a single drop of $2 \%$ fluorescein in each eye, the tear meniscus was observed under blue-filtered light 5 minutes after instillation in the upright position. A stained meniscus suggested lacrimal drainage obstruction. Next, lacrimal irrigation test was performed and classified into good passage; complete flow of fluid into nose and no reflux to lacrimal punctum, partial passage; partial flow of fluid into nose and partial reflux to lacrimal punctum and no passage; no flow of fluid into nose and complete reflux to lacrimal punctum. Diagnostic probing was performed by a 0 or 1 Bowman probe. Distal canalicular obstruction was described as either membranous stop or soft stop, as the obstruction may be a membranous block or more solid in nature. All examinations were performed by a single ophthalmologist (JP). Patients with congenital NLDO, lower lid laxity, entropion, dry eye, glaucoma, keratitis, uveitis, or a history of trauma to the nasolacrimal system or in whom insertion of the dacryoendoscope failed due to diffuse proximal canalicular obstruction were excluded from the study.

\section{Surgical instrument and procedures}

The dacryoendoscope used in this study was a Ruido Fiberscope (FiberTech, Tokyo, Japan), which is a rigid endoscope consisting of a viewing lens, fiberoptic cable, and irrigation channel. It enables the management of NLDO while allowing direct observation of the lacrimal drainage system from the punctum to the nasal opening. This instrument has a probe length of $50 \mathrm{~mm}$, outer diameter of $0.9 \mathrm{~mm}$, and $27^{\circ}$ viewing angle at $10 \mathrm{~mm}$ from the tip. Under local anesthesia with sedation, the dacryoendoscope 
was initially inserted through the upper punctum to examine the upper canaliculus, common canaliculus, lacrimal sac, and nasolacrimal duct. It was then inserted through the lower punctum to examine the lower canaliculus, in the same manner. Intraoperatively, the extent of obstruction along the NLDwas classified as normal, focal, multiple focal, or diffuse. Diffuse represented obstructions greater than $2 \mathrm{~mm}$. Saline solution was intermittently pushed through the irrigation channel to obtain a clear view of the lumen. The site and extent of obstruction in the lacrimal drainage system were examined, and recanalization was performed using the pressure by the sheath-covered dacryoendoscopic head itself and hydro pressure by performing irrigation through the irrigation channel. After recanalization, bougination was performed via repetitive movements of the dacryoendoscope. Finally, sheath-guided intubation of the silicone tube (Bika; Yowon Meditec, Wonju, Korea) was performed. It was planned that all patients would be intubated with a silicone tube for 6 months. All procedures were performed by a single surgeon (JP).

\section{Outcome measures}

After dacryoendoscopic surgery, the patients were followed up every week for the first month, every 2 weeks for the second month, and every month thereafter until 6 months postoperatively. After 6 months, they were followed up every 6 to 12 months.

At 4 weeks, 12 weeks, 6 months, and 1 year postoperatively, and at the last visit, all patients were examined to assess surgical success. Surgical outcome was categorized into complete response, partial response, and failure. Complete response was defined as the absence of epiphora, normal FDDT findings, and good fluid passage during lacrimal irrigation. Partial response was defined as normal FDDT findings and either the absence of epiphora or good passage of lacrimal irrigation. Failure was defined as abnormal FDDT findings, the presence of epiphora, and partial or no passage of lacrimal irrigation. Patients with complete and partial responses were grouped into a success group. The presence of postoperative inflammation was examined by detecting inflammatory signs such as conjunctival injection and discharge of pus around the silicone tubes and puncta by slit-lamp examinations. If the inflammation was not controllable with systemic third-generation cephalosporin and antibiotics eye drops and ointments, early removal of the silicone tubes was performed.

Factors such as patient age, preoperative lacrimal irrigation and diagnostic probing findings, the presence of dacryocystitis, the site and extent of NLDO and canalicular obstruction shown by the dacryoendoscope, early extubation of the silicone tube, and the presence of postoperative inflammation were analyzed to examine their correlation

Table 1. Demographic, preoperative, intraoperative, and postoperative characteristics of patients with obstruction in the lacrimal drainage system

\begin{tabular}{lc}
\hline Characteristic & Value \\
\hline No. of eyes (patients) & $74(51)$ \\
Male : female (no. of patients) & $14(10): 60(41)$ \\
Age (yr) & $60.3 \pm 10.0(34-80)$ \\
Lacrimal irrigation test & \\
Good passage & $0(0)$ \\
Partial passage & $49(66.2)$ \\
No passage & $25(33.8)$ \\
Preoperative diagnostic probing test & \\
Hard stop & $38(51.4)$ \\
Distal membranous stop & $19(25.7)$ \\
Distal soft stop & $17(23.0)$ \\
Dacryocystitis & $7(9.5)$ \\
Mean time to silicone tube extubation & $5.6 \pm 1.4$ \\
(mon) & \\
Postoperative follow-up period (mon) & $58(6.5-72)$ \\
DES canalicular findings & \\
Normal & $60(81.1)$ \\
Partial obstruction & $6(10.8)$ \\
Complete obstruction & $31(41.9)$ \\
DES NLDO findings & $35(47.3)$ \\
Normal & \\
Focal & $6(6.8)$ \\
Multiple focal & \\
Diffuse & \\
Canainage system (\%) & \\
Nasolacrimal duct & \\
\hline
\end{tabular}

Values are presented as mean \pm standard deviation (range), number of eyes (\%), or median (range), unless otherwise indicated.

DES = dacryoendoscopic; $\mathrm{NLDO}=$ nasolacrimal duct obstruction. 
with surgical outcome. Subjects exhibiting complete and partial responses were grouped into a success group, and we compared the aforementioned factors between the suc- cess and failure groups. Finally, Kaplan-Meier survival analysis was used to analyze the long-term success rate of the procedure according to the factors found to have statis-

Table 2. Dacryoendoscopic canalicular findings according to the preoperative diagnostic lacrimal probing findings $(\mathrm{n}=74)$

\begin{tabular}{lccc}
\hline \multirow{2}{*}{ Preoperative diagnostic lacrimal probing finding } & \multicolumn{2}{c}{ Dacryoendoscopic canalicular finding } \\
\cline { 2 - 4 } Hard stop $(\mathrm{n}=38)$ & Patent & Partial obstruction & Complete obstruction \\
Distal membranous stop $(\mathrm{n}=19)$ & 8 & 30 & 0 \\
Distal soft stop $(\mathrm{n}=17)$ & 0 & 1 & 18 \\
\hline
\end{tabular}

Values are number of eyes.

Table 3. Comparison of factors associated with surgical outcome between the success and failure groups

\begin{tabular}{|c|c|c|c|}
\hline Variable & Success proportion $(n=66)$ & Failure proportion $(\mathrm{n}=8)$ & $p$-value ${ }^{*}$ \\
\hline Patient age, mean $\pm \mathrm{SD}(\mathrm{yr})$ & $60.1 \pm 11.4$ & $62.1 \pm 6.7$ & 0.324 \\
\hline $\begin{array}{l}\text { The extent of obstruction determined by lacrimal } \\
\text { irrigation test }\end{array}$ & & & $0.010^{*}$ \\
\hline Good passage & $0(0)$ & $0(0)$ & \\
\hline Partial passage & $71.2(47)$ & $25.0(2)$ & \\
\hline No passage & $28.8(19)$ & $75.0(6)$ & \\
\hline Preoperative diagnostic probing test & & & 0.484 \\
\hline Hard stop & $51.5(34)$ & $50.0(4)$ & \\
\hline Distal membranous stop & $24.2(16)$ & $37.5(3)$ & \\
\hline Distal soft stop & $24.2(16)$ & $12.5(1)$ & \\
\hline Dacryocystitis & $9.1(6)$ & $12.5(1)$ & 0.650 \\
\hline Early silicone tube extubation & $6.1(4)$ & $25.0(2)$ & 0.247 \\
\hline Postoperative inflammation & $13.6(9)$ & $75.0(6)$ & $<0.001^{*}$ \\
\hline DES canalicular findings & & & 0.439 \\
\hline Normal & $9.1(6)$ & $25.0(2)$ & \\
\hline Partial obstruction & $40.9(27)$ & $45.0(4)$ & \\
\hline Complete obstruction & $50.0(33)$ & $25.0(2)$ & \\
\hline DES NLDO findings & & & 0.352 \\
\hline Normal & $7.6(5)$ & $12.5(1)$ & \\
\hline Focal & $33.3(22)$ & $25.0(2)$ & \\
\hline Multiple focal & $18.2(12)$ & $0(0)$ & \\
\hline Diffuse & $40.9(27)$ & $62.5(5)$ & \\
\hline $\begin{array}{l}\text { Location of obstruction in the lacrimal drainage } \\
\text { system }\end{array}$ & & & 0.407 \\
\hline Canaliculus & $7.6(5)$ & $12.5(1)$ & \\
\hline Nasolacrimal duct & $9.1(6)$ & $25.0(2)$ & \\
\hline Canaliculus and nasolacrimal duct & $83.3(55)$ & $62.5(5)$ & \\
\hline
\end{tabular}

Values are number of eyes presented as number (\%), unless otherwise indicated

$\mathrm{SD}=$ standard deviation; DES = dacryoendoscopic; NLDO = nasolacrimal duct obstruction.

*Pearson chi-square test. 
tical significance.

\section{Statistical analysis}

Statistical analysis was performed using IBM SPSS ver. 20.0 (IBM Corp., Armonk, NY, USA). To identify factors associated with surgical outcome, the Pearson chi-square test and multivariable logistic regression analysis were used. Kaplan-Meier survival analysis was used to analyze the long-term success rate of the procedure according to the factors found to be statistically significant. A $p$-value $<0.05$ was considered statistically significant.

\section{Results}

The study included 74 eyes of 51 patients (10 male patients and 41 female patients). The mean age of the patients was $60.3 \pm 10.0$ years (range, $34-80$ years). Preoperatively, by lacrimal irrigation test, no passage of the lacrimal drainage system was found in 25 eyes (33.8\%) and partial passage in $49(66.2 \%)$. Diagnostic lacrimal probing showed a hard stop in 38 eyes (51.4\%), distal membranous stop in 19 (25.7\%), and distal soft stop in 17 (23.0\%). Dacryoendoscopic examination of the canaliculi showed normal canaliculi in only eight eyes (10.8\%), stenosis in 31 (41.9\%), and obstruction in 35 (47.3\%). Dacryoendoscopic determination of the extent of obstruction in NLD showed that six eyes (6.8\%) exhibited normal NLD, 24 (32.4\%) exhibited focal obstruction, 12 (16.2\%) exhibited focal obstructions in multiple locations, and 32 (43.2\%) exhibited diffuse obstruction in NLD. Sixty eyes (81.1\%) were found to have both canalicular and NLD stenosis or obstruction. The mean time to silicone tube extubation was $5.6 \pm 1.4$ months and the median follow-up period was 58 months (range, 6.5-72 months). The demographics of the patients and findings of the dacryoendoscopic examinations are summarized in Table 1 .

Table 4. The result of multivariable logistic regression analysis of factors affecting failure of dacryoendoscopy-guided recanalization and silicone tube intubation

\begin{tabular}{|c|c|c|c|}
\hline \multirow{2}{*}{ Variable } & \multicolumn{3}{|c|}{ Surgical success } \\
\hline & Odds ratio & $95 \% \mathrm{CI}$ & $p$-value \\
\hline Postoperative inflammation (yes) & 43.900 & $1.744-1,109.716$ & $0.022^{\dagger}$ \\
\hline Age ( $>60$ years $)$ & 1.428 & $0.026-77.911$ & 0.861 \\
\hline Lacrimal irrigation test finding (no passage) & 9.481 & $0.197-456.645$ & $0.025^{\dagger}$ \\
\hline \multicolumn{4}{|l|}{ Diagnostic probing test } \\
\hline Distal membranous stop & 0.600 & $0.016-22.167$ & 0.782 \\
\hline Distal soft stop & 0.505 & $0.036-6.999$ & 0.610 \\
\hline Hard stop & Reference & & \\
\hline Dacryocystitis (yes) & 0.804 & $0.015-43.446$ & 0.914 \\
\hline \multicolumn{4}{|l|}{ DES canalicular findings } \\
\hline Partial obstruction & 0.107 & $0.002-7.025$ & 0.296 \\
\hline Complete obstruction & 0.199 & $0.005-7.369$ & 0.381 \\
\hline Normal & Reference & & \\
\hline \multicolumn{4}{|l|}{ DES NLDO findings } \\
\hline Focal obstruction & 0.041 & $0.0-6.935$ & 0.222 \\
\hline Multiple focal obstruction & 0 & 0 & 0.975 \\
\hline Diffuse obstruction & 0.126 & $0.002-7.959$ & 0.327 \\
\hline Normal & Reference & & \\
\hline
\end{tabular}

$\mathrm{CI}=$ confidence interval; DES = dacryoendoscopic; NLDO = nasolacrimal duct obstruction.

${ }^{*}$ Calculated by the analysis of logistic regression model for surgical success for patients with obstruction in lacrimal drainage system, after adjustment for sex, age, lacrimal irrigation test finding, diagnostic probing finding, presence of dacryocystitis, and DES canalicular and NLDO findings; ${ }^{\dagger}$ Statistically significant associations $(p<0.05)$. 
According to the preoperative diagnostic probing findings in canaliculus, the dacryoendoscopic examination results of the canaliculus are described in Table 2. When canaliculus was visualized by the dacryoendoscope, not all preoperative diagnostic probing findings matched the actual status of canaliculus. Among 38 eyes which showed hard stop preoperatively, only eight eyes showed normal canaliculus. Other thirty eyes showed partial obstruction in canaliculus. Among 19 eyes which showed distal membranous stop preoperatively, one eye showed partial obstruction and 18 eyes showed complete obstruction. However, all 17 eyes which showed distal soft stop preoperatively showed complete canalicular obstruction by the dacryoendscope.

At the last visit, complete response was noted in 56 eyes (75.7\%), partial response in 10 (13.5\%), and failure in eight (10.8\%), and accordingly, the success group consisted of 66 eyes (89.2\%) and the failure group consisted of eight eyes (10.8\%). Demographic, preoperative, intraoperative, and postoperative factors were compared between the success group and the failure group to identify factors associated with surgical outcome, as shown in Tables 3 and 4. The preoperative findings of lacrimal irrigation test and the presence of postoperative inflammation were associated with lower success rate ( $p=0.01$ and $p<0.001$, respectively). According to preoperative lacrimal irrigation test, a success rate of $76 \%$ in patients with no passage and $95.9 \%$ in patients with partial passage was achieved. By analysis of logistic regression model for surgical failure, the presence of postoperative inflammation increased the failure rate by 43.9 times and the finding of no passage in preoperative lacrimal irrigation test increased the failure rate by 9.4 times ( $p=0.022$ and $p=0.025$, respectively) (Table 4). Patient age, the presence of dacryocystitis, early extubation of the silicone tube, and canalicular findings were not significantly associated with surgical outcome. In addition, the extent of NLDO, which was determined via dacryoendoscopy and classified as normal, focal, multiple focal, or diffuse obstruction, was not significantly associated with surgical outcome. However, subjects with diffuse obstruction had a failure rate of $15.6 \%$, which was higher than that noted in subjects with focal or multiple focal obstructions $(p=0.352)$.

Finally, Kaplan-Meier survival analysis was used to analyze the long-term success rate according to the presence of postoperative inflammation and the preoperative lacri- mal irrigation test finding. In subjects without inflammation, the overall success rate remained at $98.0 \%$ until 33 months postoperatively when it decreased to $96.6 \%$. Failure due to postoperative inflammation was found to occur in the first 14 months after surgery, after which the success rate remained at $60.0 \%(p<0.001)$ (Fig. 1). In subjects with no passage in the preoperative lacrimal irrigation test, the success rate remained at $80.0 \%$ during the first 14 months after surgery but decreased to $76.0 \%$ at 34 months postoperatively. On the other hand, the subjects with partial passage in the preoperative lacrimal irrigation test exhibited an overall success rate of $95.9 \%$, which remained stable throughout the follow-up period ( $p=0.01$ ) (Fig. 2). Among the eight eyes in which the procedure was unsuccessful, five underwent dacryocystorhinostomy (DCR) using an endonasal endoscope and the remaining three were followed up without further treatment. No complications were observed in any patient.

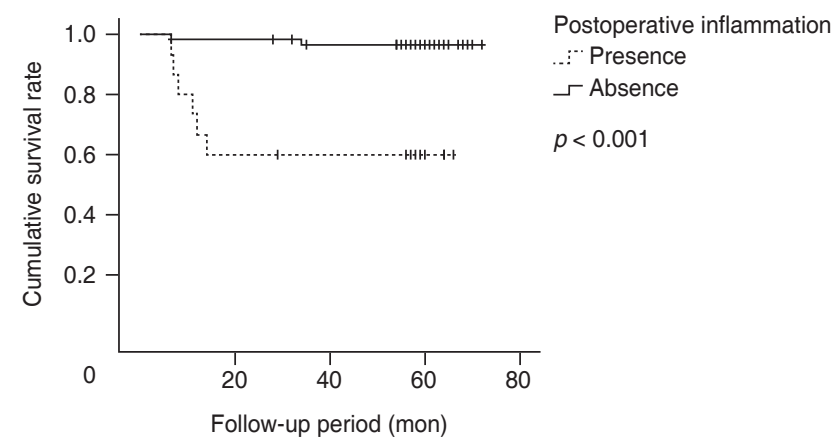

Fig. 1. Kaplan-Meier survival analysis of the success rate of dacryoendoscopic-guided recanalization and silicone tube intubation according to the presence of postoperative inflammation.

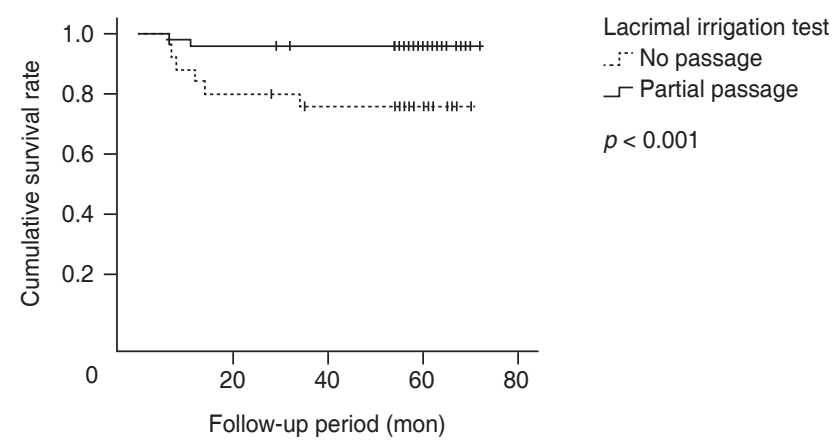

Fig. 2. Kaplan-Meier survival analysis of the success rate of dacryoendoscopic-guided recanalization and silicone tube intubation according to the extent of obstruction determined by preoperative lacrimal irrigation test. 


\section{Discussion}

Up to now, endonasal or external DCR has been the standard treatment for PNLDO. This procedure has a high success rate of approximately $90 \%$, but osseous ablation is unavoidable and copious hemorrhage can occur [13-18]. STI is an alternative treatment for PNLDO and is easier to perform than DCR. However, intubation is performed blindly and complications such as pseudotract formation can occur. The reported success rates of STI vary from approximately $50 \%$ to $86 \%$ and are likely dependent on the age of the patients and the presence of resistance in the preoperative irrigation test [19-22]. The early postoperative effect of STI is good, but relapse is commonly observed when patients are followed up long-term. A previous study reported a success rate of $77 \%$ after 6 months, $75 \%$ after 1 year, and $51 \%$ after 3 years [20]. Based on our results with a 6-year success rate of $95.9 \%$ in the eyes with preoperative lacrimal irrigation test finding of partial passage, STI with dacryoendoscopy is more effective compared to the previous conventional silicone tube insertion after probing. In cases of no passage in lacrimal irrigation test, this procedure had a lower success rate of $76 \%$. However, our study comprised a long follow-up period and included a large percentage $(81.1 \%)$ of subjects with canalicular and NLDO, so dacryoendoscopic surgery is worth trying in patients who showed no passage preoperatively in lacrimal irrigation test.

Obstruction of the lacrimal drainage system can occur focally or diffusely at one point or multiple points along the canaliculi, common canaliculus, lacrimal sac, or nasolacrimal duct. The presence of mucus, debris, stones, edema, or fibrosis can lead to narrowing or complete obstruction of the lacrimal drainage system. Conventional diagnostic tests carried out in an outpatient clinic, namely FDDT, lacrimal irrigation, diagnostic probing, dacryocystography, and dacryoscintigraphy, can only indirectly determine the site and extent of obstruction. On the other hand, dacryoendocopy enables the determination of the exact site and extent of obstruction anywhere along the lacrimal drainage system by allowing direct visualization of the passage.

Sasaki et al. [23] used a dacryoendoscope and nasal endoscope to determine the site of obstruction in patients with PNLDO. They reported that $73.2 \%$ of cases exhibited obstruction at the sac/duct junction, termed higher NLDO, while $26.8 \%$ of cases exhibited obstruction at the ostium of the nasolacrimal duct, termed lower NLDO. They treated the patients with lower NLDO with a newly developed technique called inferior meatal dacryorhinotomy, which involves intubation of a silicone tube assisted by a dacryoendoscope, nasal endoscope, and radio-frequency scalpel, and osseous ablation was not needed. They reported a 2 -year success rate of $87 \%$ [24]. The aforementioned authors demonstrated that a dacryoendoscope can be used to identify the exact location of obstruction and proved that lower NLDO can be treated without osseous ablation. The cause of NLDO, typically debris, mucus, stones, or narrowing, can also be visualized using a dacryoendoscope, and removal of the source of obstruction and recanalization can restore the functioning of the lacrimal drainage system.

In this study, preoperative diagnostic lacrimal probing showed a hard stop in 38 eyes (51.4\%), distal membranous stop in 19 (25.7\%), and distal soft stop in 17 (23.0\%). However, dacryoendoscopic examination showed that only eight eyes (10.8\%) exhibited normal canaliculi, whereas 66 $(89.2 \%)$ were found to have canalicular stenosis or obstruction. The reason for this difference in canalicular findings might be that preoperative diagnostic lacrimal probing was carried out mainly through the lower punctum into the lower canaliculus, while dacryoendoscopic examination was performed in both canaliculi. However, this cannot single-handedly explain the large discrepancy noted. The substantial discrepancy between the proportion of eyes with abnormal canaliculi and the proportion of eyes which exhibited hard stops preoperatively implies that the ability of diagnostic lacrimal probing to evaluate canalicular obstruction is limited. When direct dacryoendoscopic examination is performed, it is possible that canalicular stenosis can exist to some extent.

One of the causes of NLDO recurrence after DCR is obstruction of the canaliculi or common canaliculus. DCR involves the creation of a passage between the lacrimal sac and nasal mucosa. This technique allows the surgeon to manage lacrimal sac and NLDO, but recanalizing canalicular obstructions is more difficult. Canalicular obstructions are forcefully trephined, which can damage the mucosa or promote pseudo tract formation. However, stenosis or obstruction of the canaliculi and common canaliculus can be not only identified but also managed by dacryoendscopic-guided recanalization, which should significantly lower 
the recurrence rate.

In the study, dacryolith was found in seven eyes during the operation and all had complete response. We believe that the presence of dacryolith can be a predictive factor for successful dacryoendoscopy-guided STI and our result is in accordance with previous studies $[25,26]$. Choi et al. [26] reported a success rate of $91.8 \%$ in eyes with dacryolith as compared to $80.5 \%$ in nondacryolith group and explained that a dacryolith is related to acute or subacute inflammation rather than chronic inflammation or fibrosis.

Among the eyes which had postoperative inflammation, the average age of the failure group was 62.3 years, whereas that of the success group was 55.8 years. Although statistically insignificant, age can be an important factor that can affect the occurrence of inflammation and its control. In addition, among six eyes in the failure group which occurred postoperative inflammation, three had comorbid systemic or ocular diseases. One had thyroid eye disease, the other had gastric cancer and a history of chemotherapy, and another had cicatricial entropion in the lower eyelid due to blepharoplasty. In the case of systemic or ocular diseases which can induce inflammation, special postoperative care is needed. For the postoperative inflammation, systemic and topical antibiotics and anti-inflammation can help to control the inflammation. And if the inflammation is not controlled, removal of silicone tube should be performed as silicone tube itself can induce inflammatory reaction and stimulation of granulation tissue.

Treatment of NLDO should be decided on the basis of the location and extent of obstruction. We tentatively suggest that NLDO is managed initially by dacryoendoscopy-guided recanalization and STI, which is a less invasive procedure than DCR. When failure or relapse occurs, DCR should be considered the second-choice treatment for complete and diffuse obstruction. In cases of partial NLDO, the dacryoendoscopic procedure should be reattempted. Conversely, in cases of DCR failure, dacryoendoscopy can be used to examine the canaliculi and search for obstruction or adhesions between the remnant lacrimal sac mucosa and scar tissue.

In conclusion, dacryoendoscope-guided recanalization and STI is an effective treatment for NLDO, especially those with partial NLDO. The use of a dacryoendoscope facilitates the determination of the precise site of obstruction, and STI can be performed safely in patients. Most importantly, postoperative management to control inflam- mation is important to maximize treatment success.

Conflicts of Interest: None.

Acknowledgements: None.

Funding: None.

\section{References}

1. Emmerich KH, Meyer-Rusenberg HW, Simko P. Endoscopy of the lacrimal ducts. Ophthalmologe 1997;94:732-5.

2. Mullner K, Bodner E, Mannor GE. Endoscopy of the lacrimal system. Br J Ophthalmol 1999;83:949-52.

3. Ali MJ. Dacryoendoscopic examination of the lacrimal system. In: Ali MJ, editor. Principles and practice of lacrimal surgery. 2nd ed. Singapore: Springer; 2018. p. 103-10.

4. Ali MJ, Paulsen F. Human lacrimal drainage system reconstruction, recanalization, and regeneration. Curr Eye Res 2020;45:241-52.

5. Matsumura N, Suzuki T, Goto S, et al. Transcanalicular endoscopic primary dacryoplasty for congenital nasolacrimal duct obstruction. Eye (Lond) 2019;33:1008-13.

6. Emmerich KH, Steinhauer J, Meyer-Rusenberg HW, Luchtenberg M. Dacryoendoscopy: current status. Ophthalmologe 1998;95:820-2.

7. Sasaki T, Sounou T, Sugiyama K. Dacryoendoscopic surgery and tube insertion in patients with common canalicular obstruction and ductal stenosis as a frequent complication. Jpn J Ophthalmol 2009;53:145-50.

8. Fujimoto M, Ogino K, Matsuyama H, Miyazaki C. Success rates of dacryoendoscopy-guided probing for recalcitrant congenital nasolacrimal duct obstruction. Jpn J Ophthalmol 2016;60:274-9.

9. Al Kadah B, Wolf G, Schick B. Lacrimal systems endoscopy with a new endoscope system. Laryngorhinootologie 2010;89:730-6.

10. Singh S, Ali MJ. A review of diagnostic and therapeutic dacryoendoscopy. Ophthalmic Plast Reconstr Surg 2019;35: 519-24.

11. Lee SM, Chung SJ, Lew H. Clinical efficacy of lacrimal endoscopy assisted silicone tube intubation in patients with nasolacrimal duct obstruction. J Korean Ophthalmol Soc 2018;59:582-8.

12. Volkerling A, Struck HG, Kunert KS, et al. Dacryoendoscopic surgery-factors influencing postoperative results. Klin Monbl Augenheilkd 2018;235:24-30. 
13. Kong YT, Kim TI, Kong BW. A report of 131 cases of endoscopic laser lacrimal surgery. Ophthalmology 1994;101: 1793-800.

14. Hartikainen J, Antila J, Varpula M, et al. Prospective randomized comparison of endonasal endoscopic dacryocystorhinostomy and external dacryocystorhinostomy. Laryngoscope 1998;108:1861-6.

15. Tarbet KJ, Custer PL. External dacryocystorhinostomy. Surgical success, patient satisfaction, and economic cost. Ophthalmology 1995;102:1065-70.

16. Becker BB. Dacryocystorhinostomy without flaps. Ophthalmic Surg 1988;19:419-27.

17. Jung SK, Kim YC, Cho WK, et al. Surgical outcomes of endoscopic dacryocystorhinostomy: analysis of 1083 consecutive cases. Can J Ophthalmol 2015;50:466-70.

18. Bartley GB, Nichols WL. Hemorrhage associated with dacryocystorhinostomy and the adjunctive use of desmopressin in selected patients. Ophthalmology 1991;98:1864-6.

19. Han JS, Park IK, Shin JH. Success rate of silicone intubation between nasolacrimal duct obstruction and stenosis according to dacryocystography. J Korean Ophthalmol Soc 2013;54:845-9.

20. Kwon YH, Lee YJ. Long-term results of silicone tube intubation in incomplete nasolacrimal duct obstruction
(NLDO). J Korean Ophthalmol Soc 2008;49:190-4.

21. Bleyen I, van den Bosch WA, Bockholts D, et al. Silicone intubation with or without balloon dacryocystoplasty in acquired partial nasolacrimal duct obstruction. Am J Ophthalmol 2007;144:776-80.

22. Fulcher T, O'Connor M, Moriarty P. Nasolacrimal intubation in adults. Br J Ophthalmol 1998;82:1039-41.

23. Sasaki T, Nagata Y, Sugiyama K. Nasolacrimal duct obstruction classified by dacryoendoscopy and treated with inferior meatal dacryorhinotomy. Part I: positional diagnosis of primary nasolacrimal duct obstruction with dacryoendoscope. Am J Ophthalmol 2005;140:1065-9.

24. Sasaki T, Nagata Y, Sugiyama K. Nasolacrimal duct obstruction classified by dacryoendoscopy and treated with inferior meatal dacryorhinotomy: part II. Inferior meatal dacryorhinotomy. Am J Ophthalmol 2005;140:1070-4.

25. Kominek P, Cervenka S, Zelenik K, et al. Lacrimal sac dacryolith (76 cases): a predictive factor for successful endonasal dacryocystorhinostomy? Eur Arch Otorhinolaryngol 2014;271:1595-9.

26. Choi SW, Park JS, Kwon AY, Lew H. Clinicopathological study of lacrimal sac dacryolith in patients treated for nasolacrimal duct obstruction. J Korean Ophthalmol Soc 2016;57:6-13. 